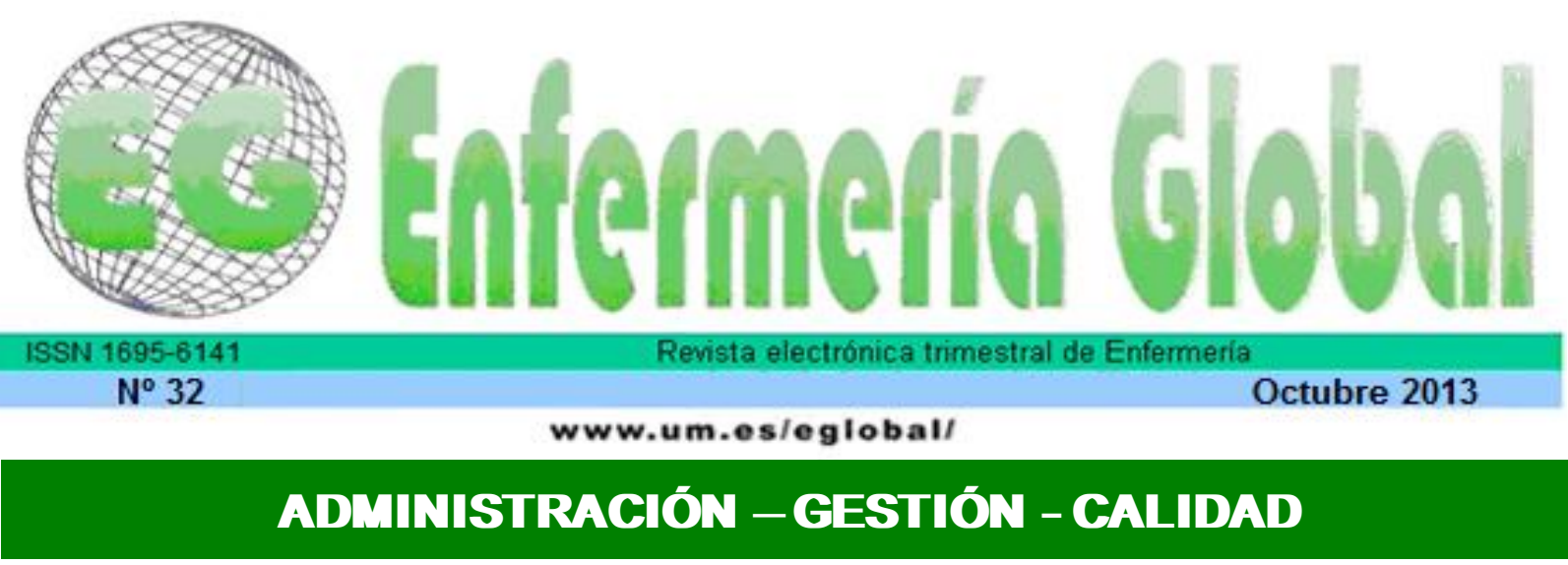

\title{
La muerte y el proceso de morir: sentimientos manifestados por los enfermeros
}

A morte e o processo de morrer: sentimentos manifestados por enfermeiros

Death and the dying process: feelings expressed by nurses

\section{*Souza e Souza, Luis Paulo **Mota Ribeiro, Juliana **Barbosa Rosa, Renata ${ }^{* * *}$ Ribeiro Gonçalves, Renata Cristina ${ }^{* * * *}$ Oliveira e Silva, Carla Silvana *****Barbosa, Dulce Aparecida}

*Graduando en Enfermería. E-mail: luis.pauloss@hotmail.com **Enfermera ***Enfermera, Profesora de la Facultad de Salud y Desarrollo Humano Santo Agostinho de Montes Claros.**** Enfermera, Doctoranda en Ciencias por la UNIFESP. Profesora de la Universidad Estadual de Montes Claros y de la Facultad de Salud y Desarrollo Humano Santo Agostinho. *****Pos Doctora en Ciencias. Profesora Libre docente y Asociada de la Escuela Paulista de Enfermería. Universidad Federal de São Paulo - UNIFESP. São Paulo, Brasil.

\section{Palabras clave: Muerte; actitud frente a la muerte; enfermería; Unidades de cuidados Intensivos Palavras chave: Morte; atitude frente à morte; enfermagem; Unidades de Terapia Intensiva Keywords: Death; Attitude to death; Nursing; Intensive Care Units.}

\section{RESUMEN}

El estudio pretende conocer los sentimientos experimentados por los enfermeros frente a la muerte y el proceso de morir. Investigación cualitativa, realizada con siete enfermeros de una UCI de un hospital en Montes Claros, Minas Gerais. Se utilizó entrevista no estructurada para la recolección de datos, que se analizaron mediante la técnica de análisis de contenido. La confrontación de los encuestados frente a la muerte está impregnada de sentimientos tales como: impotencia, angustia, sufrimiento, miedo, que interfieron en la asistencia prestada al enfermo y a su familia. Por otro lado, a pesar de la vivencia constante con la muerte, las enfermeras aún se sensibilizan con el proceso de muerte.

\section{RESUMO}

O estudo objetivou conhecer os sentimentos vivenciados pelos enfermeiros diante da morte e o processo de morrer. Pesquisa qualitativa, realizada com sete enfermeiros de uma UTI de um hospital em Montes Claros, Minas Gerais. Utilizou-se entrevista não estruturada para coleta dos dados, os quais foram analisados utilizando a técnica de análise do conteúdo. O enfrentamento dos entrevistados 
diante da morte é permeado por sentimentos como: impotência, angústia, sofrimento, medo, os quais interferiram na assistência prestada ao enfermo e sua família. Por outro lado, apesar da vivência constante com a morte, os enfermeiros ainda se sensibilizam com o processo de morte.

\section{ABSTRACT}

The study aimed to know the feelings experienced by nurses in the face of death and dying process. Qualitative research, conducted with seven nurses of a UTI from a hospital in Montes Claros, Minas Gerais. Unstructured interview was used for data collection, which were analysed using the technique of content analysis. The confrontation of respondents in the face of death is permeated by feelings such as: impotence, distress, suffering, fear, which interfered in the assistance provided to the patient and his family. On the other hand, despite the constant experience with death, the nurses still raise with the process of death.

\section{INTRODUCCIÓN}

Hablar sobre la muerte, abstracta o específica, es hablar de lo que se está haciendo, de lo que no se hizo, de planes, sueños, pérdidas, del tiempo que se fue, de lo que resta aún. La muerte del otro es un recuerdo de la propia muerte, y en esto consiste la dificultad de las personas en dar a aquellos que fallecen la ayuda y el afecto que necesitan, al despedirse de los demás ${ }^{(1)}$.

Respecto a la habilidad en lidiar con el proceso de la muerte y el morir, la diferencia básica entre legos y profesionales de la salud es que en la vida de estos, la muerte forma parte de lo cotidiano y puede tornarse su compañera de trabajo diario. Sin embargo, percibir la muerte puede constituir un acceso para el conocimiento sobre la muerte y el morir ${ }^{(2)}$.

Frecuentemente los profesionales de la salud, específicamente los enfermeros, están expuestos a casos de enfrentamiento de la muerte de personas bajo su cuidado, encontrando dificultades en encararla como parte integrante de la vida, teniéndola como resultado del fracaso terapéutico y del esfuerzo por la cura. Estudiar las concepciones culturales del proceso salud-enfermedad-muerte en las diferentes sociedades puede proporcionar una posibilidad de comprensión a los profesionales de enfermería de sus propios valores y creencias ante la muerte y del proceso de morir, así como también sus actitudes y acciones relacionadas con las cuestiones de lo cotidiano que influyen en la vida personal y profesional ${ }^{(3)}$.

La simple convivencia diaria con la muerte, no impide a los profesionales la expresión de malos sentimientos, por el contrario, es necesario que tengan mejor comprensión sobre este hecho, para que puedan sufrir menos, controlar las emociones y ayudar mejor a los pacientes y a sus familiares, a pesar de que la muerte forma parte de la vida y sea exactamente esta perspectiva la que va replantear la propia vida, hablar sobre el tema siempre asustó al ser humano, aun tratándose de profesionales de la salud, conscientes de la impotencia humana y de la propia muerte ${ }^{(4)}$.

Ante esta constatación, surge, entonces, la necesidad de los enfermeros de romper el silencio y atreverse a hablar de sus dolores, miedos, del luto que debe ser elaborado, a fin de que sus demandas sean atendidas y, consecuentemente, los cuidados sean mejor brindados. Es importante que ellos permitan entristecerse y no se sientan culpables ${ }^{(1,5)}$. Ante este contexto, vino el siguiente cuestionamiento: ¿Cuáles son los sentimientos vividos por los Profesionales Enfermeros frente a la muerte y el proceso 
de morir?

La muerte significa, normalmente, dolor y soledad para los que quedan. Por lo tanto, desde esta perspectiva, no es solo la destrucción de un estado físico y biológico que ella trae, sino también el fin de un ser en correlación con el otro ${ }^{(6)}$.

Así, este estudio tuvo como objetivo conocer los sentimientos experimentados por enfermeros ante la muerte y el proceso de morir.

\section{METODOLOGÍA}

Se trata de un estudio cualitativo, de carácter exploratorio-descriptivo. Participaron del estudio enfermeros que actuaban en la Unidad de Cuidados Intensivos (UCI) de un Hospital Filantrópico en Montes Claros, norte de Minas Gerais, Brasil.

Como criterio de inclusión, se tuvo en cuenta el hecho de haber experimentado la muerte en el empleo actual y el deseo en participar del estudio. Así, se totalizaron siete enfermeros entrevistados. Para la recolección de datos, se utilizó entrevista no estructurada, abordando aspectos inherentes a la vida profesional al lidiar con la muerte y el proceso de morir.

Las entrevistas fueron realizadas en la propia $\mathrm{UCl}$, en el mes de mayo de 2011 , individualmente, con fecha y horario programado de acuerdo con la disponibilidad de los participantes. Las palabras fueron grabadas y transcritas en su integridad para preservar las expresiones de lenguaje utilizados por los colaboradores. Después de la transcripción, se realizó el análisis de los datos a través de la técnica de análisis de contenido (7). Para garantizar el secreto de las identidades de los entrevistados, utilizamos nombres ficticios, para la presentación de pequeños pasajes de las declaraciones.

Se resalta que todos los entrevistados firmaron el Término de Consentimiento Libre y Esclarecido y la investigación fue aprobada por el Comité de Ética en Investigación de la Asociación Educativa del Brasil - SOEBRAS- por medio del parecer CAAE 0044.0.0.445.000-11/SISNEP.

\section{RESULTADOS Y DISCUSIÓN}

La circunstancia de óbito en el ambiente hospitalario, situación en la cual se da la materialización del proceso de morir y de la muerte es, ciertamente, una experiencia impregnada de significaciones científicas, como también sociales, culturales y principalmente subjetivas ${ }^{(8)}$.

A partir del análisis de las palabras, emergieron las siguientes categorías: "Concepto de muerte"; "Sentimientos con relación a la muerte en su lugar de trabajo": "Reacciones ante la muerte y del proceso de morir", las cuales serán discutidas a continuación.

\section{Concepto de muerte}

Antes de hablar sobre la muerte, es necesario conceptuarla. La pregunta "¿qué es la muerte?" tiene múltiples respuestas y ninguna de ellas concluyente, pues la cuestión transciende los aspectos naturales o materialistas y hasta, biológicamente, es difícil 
una respuesta unánime ${ }^{(9)}$.

Autores afirman que morir, científicamente, es dejar de existir. Es cuando el cuerpo tiene colapso de sus órganos vitales, teniendo una parada progresiva de toda actividad del organismo, pudiendo ser de forma súbita, enfermedades agudas o accidentes, o lentas, enfermedades crónico-degenerativas, seguidas de una degeneración de los tejidos ${ }^{(9)}$.

"Es el tiempo que la persona ya cumplió aquí en la tierra y va vivir en otro espacio, otro lugar diferente de aquí, humana". (Luciana).

"La muerte para mí es un fenómeno natural que hoy es muy discutida, entre las religiones." (Fabiana)

"La muerte es un momento de transición, yo creo que después hay un lugar especial guardado para cada uno." (Fabiola).

Las palabras de los entrevistados muestran que la muerte es comprendida como una etapa natural, que forma parte de la vida humana y que tiene connotaciones diferentes, debido a aspectos individuales y religiosos de los entrevistados. Además se descubrió que existe un temor, un tabú al hablar sobre la muerte para algunos profesionales.

Autores aclaran que, muchas veces, los profesionales crean un mecanismo de defensa, de mantenerse a distancia, manifestar comportamiento de frialdad con relación a las situaciones, así como aparente equilibrio, en la tentativa de manejar de forma más adecuada la situación ${ }^{(1)}$.

\section{Sentimientos con relación a la muerte en su lugar de trabajo}

Es sabido que los profesionales de enfermería se encuentran más cercanos a los pacientes y están en contacto permanente con las personas enfermas y en la Unidad de Cuidados Intensivos, esto se da con más intensidad.

Los enfermeros relataron que la proximidad de la muerte de un paciente, al cual dedicó horas de trabajo, puede despertar sentimientos como impotencia, tristeza, temor y sufrimiento.

\section{Tristeza}

"Yo siento esa tristeza, ese dolor, ese malestar yo siento con relación al que queda y no con quien se va, quien se va para mi es más tranquilo." (Fabricia).

\section{Impotencia}

"(...) sin embargo, no hay ninguna muerte que ocurra que la gente no se pare y piense; ¿será lo que podríamos haber hecho o no?” (Fabiola).

\section{Sufrimiento}

"Nosotros intentamos no sufrir mucho, porque esta cuestión es difícil más aún aquí en la UCI donde nosotros tenemos óbitos casi todos los días, y nosotros 
tenemos que estar intentando crear medios para que no suframos tanto, caso contrario nosotros también quedamos muy sensibles y eso es muy malo para nosotros como profesionales." (Amanda).

Miedo

"Yo veo aquí al paciente morir, ayudo, saco los dispositivos, pero si es para mi ir en la caja, en el velatorio, yo no miro, yo no puedo mirar." (Luciana).

Nótese que otra preocupación mayor de los enfermeros es con aquellos que quedaron, con la familia. El enfrentamiento de la muerte es difícil y angustiante para el que lo vive, pudiendo ser aún más para quien lo observa, porque la muerte provoca profundas rupturas entre quien murió y el otro que continúa viviendo ${ }^{(5)}$.

Es notoria la dificultad del enfermero en hablar sobre el asunto. No obstante, las pausas en algunas palabras, el cambio en la mirada, la voz entrecortada expresan la impotencia y la falta de preparación para lidiar con la muerte.

Es relevante identificar los sentimientos vividos por los enfermeros en la práctica de la asistencia en la muerte y en el proceso de morir, pues es sabido que el autoconocimiento es un proceso importante a ser explorado, a fin de lidiar mejor con situaciones que impliquen manifestaciones profundas de emociones y en ese caso, la muerte $^{(10)}$.

\section{Reacciones ante la muerte y del proceso de morir}

Desde la formación, el profesional enfermero se siente comprometido con la vida, y es para la preservación de este que deberá sentirse capacitado. Su formación académica está fundamentada en la cura, y en ella está su mayor gratificación. Así, cuando en su cotidiano de trabajo necesita lidiar con la muerte, en general, se siente sin preparacióno, y tiende a alejarse de ella ${ }^{(11)}$.

De acuerdo con las entrevistas, los profesionales intentan, de alguna forma, mantener un distanciamiento del paciente y de su familia para disminuir la posibilidad de establecer un vínculo afectivo, como si eso pudiese disminuir su sufrimiento en el momento en que la muerte acomete a aquel paciente, como se observa en las palabras que siguen:

"A veces hasta evito un poco la familia porque cuando la familia descubre que yo soy la supervisora del sector, la familia se acerca a mí, vienen a buscarme, entonces me conoce por mi nombre, sostiene mi mano, habla conmigo y eso me arrasa, sabes." (Amanda).

"La familia termina trayendo un poco de la historia de ese paciente para nosotros, algunos traen fotos y todo, así termina en que nosotros sufrimos mucho." (Carla)

"Yo aún me emociono mucho con la muerte, principalmente aquellos casos de los pacientes con el cual nosotros nos apegamos mucho con la familia." (Luciana) 
"A veces sufrimos más con la familia que con el propio paciente que está muriéndose" (Fabiola)

El sufrimiento de los enfermeros parece estar marcado por el cumplimiento de las rutinas. Esos sufrimientos derivados del desarrollo emocional son hechos vividos en la unidad hospitalaria y están directamente ligados a los valores personales, la historia de vida y la patología que acomete al paciente. La muerte asumirá el papel de "descanso y alivio" del sufrimiento o aun de "tragedia", diferentemente de lo que juzga el sentido común de "frialdad" sobre los hechos tristes que suceden en el día a día del hospital, ya que esos trabajadores son "gente cuidando de gente" (12).

"Cuando ves a la familia llegando y tienes que ir allí juntamente con el médico a dar la noticia de que aquella persona murió, su hijo, su madre, su hermano es muy difícil, porque nosotros no sabemos qué decir a aquella persona?" (Carla).

"Y, a veces, nosotros nos colocamos en el lugar de aquella persona, hay veces en que nosotros hasta lloramos dependiendo del caso." (Fabiola).

Estudiosos dan a conocer que la muerte provoca un sentimiento de pérdida y, al mismo tiempo, un sentimiento de confort, a pesar de sentir que la vida se está yendo, sin embargo aquella persona quedará libre de sus sufrimientos. Enfrentar ese proceso es no huir de ninguna situación; es no conseguir disculpas para no estar presente en el momento de la muerte, cuando suceden situaciones consideradas no agradables, está permitido llorar, entristecerse, sentir lo que viene de adentro (13). Los profesionales de la salud terminan creando mecanismos de defensa que los auxilian en el enfrentamiento de la muerte y del proceso de morir. Porque están preparados para el mantenimiento de la vida, la muerte y el morir en su cotidiano suscita sentimientos de frustración, tristeza, pérdida, impotencia, estrés y culpa. Por lo general, la falta de preparación lleva al profesional a alejarse de la situación ${ }^{(14)}$.

El surgimiento de angustia, frustración, miedo y la falta de preparación de algunos enfermeros en lidiar con la muerte es mencionado como una falla de la enseñanza en la graduación, que no prepara a esos profesionales para la ardua rutina de los hospitales, que es vivir en común con el sufrimiento ajeno ${ }^{(15) . ~ E s e ~ s e n t i m i e n t o ~ d e ~}$ temor lleva a la reflexión, ya que el enfermero crea mecanismos y formas para vivir la situación del proceso de muerte y morir que puedan ser positivas o negativas, tanto para sí mismo, como en relación con los demás.

\section{CONCLUSIÓN}

Se ha verificado que en el enfrentamiento de la muerte y el proceso de morir por el enfermero emergen sentimientos como: impotencia, angustia, sufrimiento, tristeza, miedo. $Y$ esos sentimientos interfirieron en la asistencia ofrecida al enfermero y su familia, sin embargo, por otro lado, mostró que, a pesar de la vivencia constante con la muerte en su práctica en la $\mathrm{UCl}$, los enfermeros aun se sensibilizan con el proceso de la muerte. Se ha observado, también, una preocupación y solidaridad constante de esos profesionales con relación a la familia del paciente, siendo esta valorada e incluida en el contexto hospitalario y de cuidado.

En sus relatos, los entrevistados dijeron ver a la muerte como un hecho natural, sin embargo terminan desarrollando una forma de defensa ante el dolor y el sufrimiento. 
Pera ellos, esos "distanciamientos" son necesarios a fin de evitar daños en los aspectos psicológicos y emocionales.

Se ha observado una contradicción en las palabras de algunos profesionales, pues, a pesar de mostrarse indiferentes con la muerte del otro, mostraron también que es imposible cuidar de alguien sin involucrarse. El modo de separar el sentimiento de lo cotidiano del trabajo consiste en la habilidad de reconocer sus propios sentimientos.

De este modo, cabe resaltar la necesidad de construir alternativas para que esos enfermeros sean estimulados a pensar, discutir y comprender mejor a la muerte y el proceso de morir. Se ha comprendido que este tema debe ser más debatido en la formación académica, dando énfasis en cuestiones ligadas a la emoción, ya que es primordial que el profesional de la salud revise sus conceptos sobre la existencia, pues, si no lo hiciere, permanecerá encarando a la muerte de los pacientes como fracaso, impotencia y frustración.

\section{REFERENCIAS}

1. Aguiar IR, Veloso TMC, Pinheiro AKB, Ximenes LB. O envolvimento do enfermeiro no processo de morrer de bebês internados em Unidade Neonatal. Acta paul enferm. 2006;19(2):131-137.

2. Borges MS, Mendes N. Representações de profissionais de saúde sobre a morte e o processo de morrer. Rev bras enferm. 2012; 65(2): 324-331.

3. Gutierrez BAO, Ciampone MHT. Profissionais de Enfermagem Frente ao Processo de Morte em Unidade de Terapia Intensiva. Acta paul enferm. 2006;19(4):456-461.

4. Sanches PG, Carvalho MDB. Vivência dos enfermeiros de unidade de terapia intensiva frente à morte e o morrer. Rev gaúch enferm. 2009;30(2):289-296.

5. Carvalho LS, Silva CA, Santos ACPO, Oliveira MA, Portela SC, Regebe CMC. Percepções de morte e morrer na ótica de acadêmicos de enfermagem: estudo qualitativo. Online Braz J Nurs [periódico na internet]. 2006 [acesso em 2009 jan 17];5(3). Disponível em: http://www.uff.br/objnursing/index.php/nursing/article/view/507/116.

6. Silva Júnior FJG, Santos LCS, Moura PVS, Melo BMS, Monteiro CFS. Processo de morte e morrer: evidências da literatura científica de enfermagem. Rev bras enferm. 2011; 64(6):1122-1126.

7. Bardin L. Análise de conteúdo. Lisboa: Edições 70; 2009.

8. Nascimento $C A$, Silva $A B$, Silva $M C$, Pereira $M H$. A significação do óbito hospitalar para enfermeiros e médicos. Rev RENE. 2006; 7(1):52-60.

9. Moreira AC, Lisboa MTL. A Morte - Entre o Público e o Privado: reflexões para a prática profissional de enfermagem. Rev enferm UERJ. 2006; 14(3):447-454.

10. Oliveira WIA, Amorin RC. A Morte e o Morrer no processo de formação do Enfermeiro. Rev Gaúcha Enferm. 2008; 2(3):191-8.

11. Lunardi Filho WD, Sulzbach RC, Nunes AC, Lunardi VL. Percepções e condutas dos profissionais de enfermagem frente ao processo de morrer e morte. Texto Contex enferm. 2011;10(3):60-81.

12. Rodrigues IG, Zago MM. A morte e o morrer: maior desafio de uma equipe de cuidados paliativos. Cienc Cuid Saude. 2012; 11(suplem.):31-38.

13. Fernandes MEN, Fernandes AFC, Albuquerque ALP, Mota MLS. A morte em unidade de Terapia Intensiva: Percepções dos enfermeiros. Rev RENE. 2006; $7(1): 43-51$.

14. Susaki TT, Silva MJP, Possasi JF. Identificação das fases do processo de morrer pelos profissionais de Enfermagem. Acta paul enferm. 2006; 19(2):144-149. 
15. Costa JC, Lima RAG. Luto da equipe: revelações dos profissionais de enfermagem sobre o cuidado à criança/adolescente no processo de morte e morrer. Rev LatinoAm Enferm [Internet]. 2005 Abr [acesso em 2012 jun 18]; 13(2): 151-157. Disponível em: http://www.scielo.br/scielo.php?script=sci arttext\&pid=S0104$11692005000200004 \& \operatorname{lng}=$ en. 